\title{
Unusual polymorphs of thymine
}

Susanta Kumar Nayak ${ }^{1}$, Ramanaiah Chennuru², Prakash Muthudoss ${ }^{2}$, Srividya Ramakrishnan², Amjad B. Mohammad², R. Ravi Chandra Babu $^{3}$, Sudarshan Mahapatra ${ }^{2}$

${ }^{1}$ Department Of Chemistry, Visvesvaraya National Institute Of Technology(VNIT), Nagpur, India, ${ }^{2}$ Integrated Product Development (IPDO), Dr. Reddy's Laboratories Ltd., Bachupally, Hyderabad, India, ${ }^{3}$ Department Of Chemistry, College Of Science, GITAM University, Visakhapatnam, India

E-mail: nksusa@gmail.com

Adenine and thymine are of the most important organic molecules of life as it forms an integral part of DNA, RNA, ATP, and ADP. The double helix of DNA is built based on pairs of hydrogen bonded purines and pyrimidines as AT and GC base pairs stacked along the helix axis. However, in absence of detailed structural analysis, new interesting structural insight will pave the way to understand the mechanism and to improve their activity of drugs and design new materials of choice. In this context, we have already demonstrated the crystal structure of anhydrous adenine, which has been determined using a single crystal grown by a unique approach using a sublimation process. Further, our studies shows that the cocrystallization of free base (A-T) pair which evidences the Hoogsteen and Watson-Crick type interactions but only with respect to the adenine unit. The absence of a clear-cut Watson-Crick base pair raises intriguing questions about the basis of the DNA double helix. In this presentation, our current study will highlights the two new polymorphic forms (Form-R2 and R4) of anhydrous thymine, which are observed and unequivocally established considering different solid state and in-silico techniques. The supramolecular assembly in Form-R2 is a sheet of hydrogen bonded network similar to that found in the crystal structures of other reported anhydrous form of thymine (Form-R1). Polymorphism in thymine appears to be induced by heat and pressure. The similarity in intermolecular interactions and packing between Form-R1 and Form-R2 leads to similar thermal behavior. The transformation among the anhydrous thymine forms (Form-R1, R2 and R4) and monohydrated thymine form (Form-R3) were established via heating, sublimation and slurry in $10 \%$ ethanol methods.

[1] Mahapatra, S. Nayak, S. K. Prathapa S. J. \& Guru Row, T. N. (2008) Cryst. Growth Des. 8, 1223-1225.

[2] Chandrasekhar, S. Naik, T. R. R. Nayak, S. K. \& Guru Row, T. N. (2010). Biorg. Med. Chem. Lett. 20, $3530-3533$.

[3] Chennuru, R. Muthudoss, P. Ramakrishnan, S. Mohammad, A. B. Chandra Babu, R. R. Mahapatra, S. \& Nayak, S. K. (2016) J. Mol. Struct. 1120, 86-89.

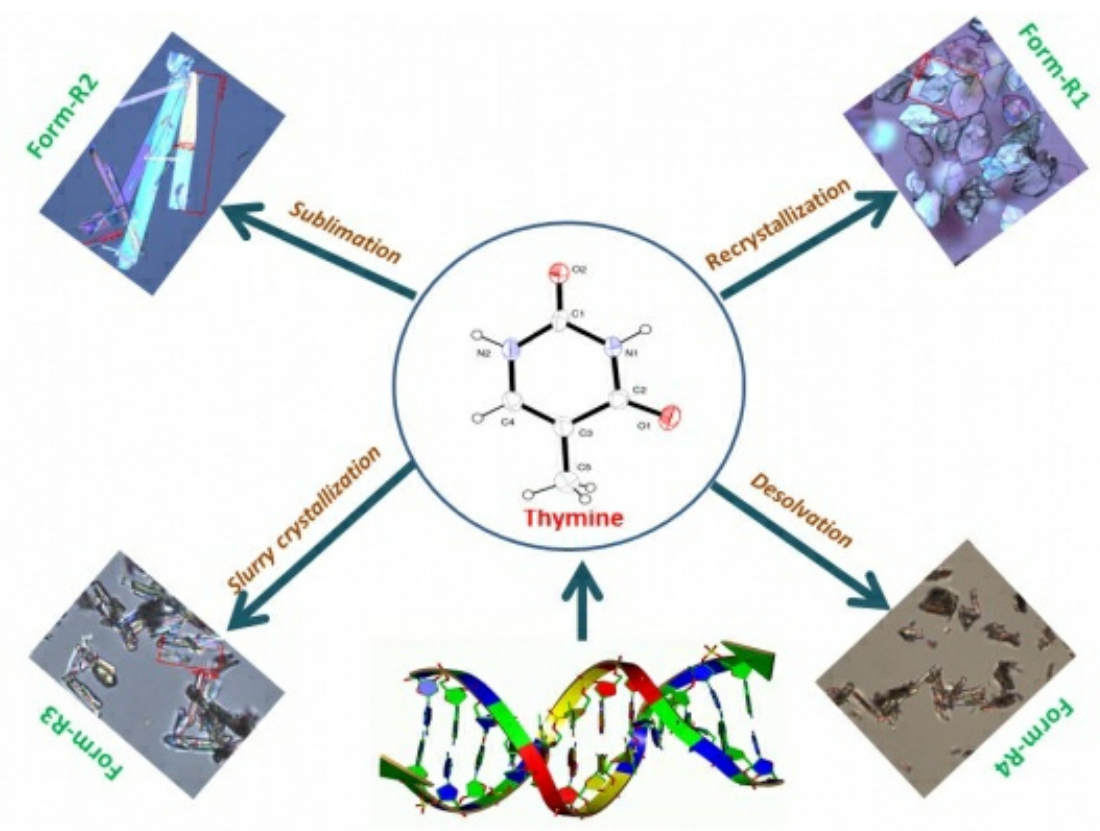

Keywords: polymorphism, thymine, nucleobase 